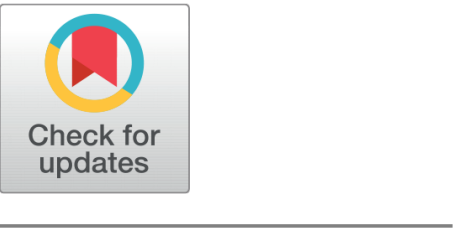

G OPEN ACCESS

Received: 02.10 .2021

Accepted: 21.01.2022

Published: 16.02 .2022

Citation: Kumar YGP, Kariyappa BS, Kurian MZ (2022) Design,

Implementation and Performance Analysis of Test Pattern Generator for Built-In Self-Test using m-GDI

Technology. Indian Journal of Science and Technology 15(5):

221-226. https://doi.org/

10.17485/IJST/v15i5.1846

* Corresponding author.

praviraj.star@gmail.com

Funding: None

Competing Interests: None

Copyright: ( $) 2022$ Kumar et al. This is an open access article distributed under the terms of the Creative Commons Attribution License, which permits unrestricted use, distribution, and reproduction in any medium, provided the original author and source are credited.

Published By Indian Society for Education and Environment (iSee)

ISSN

Print: 0974-6846

Electronic: 0974-5645

\section{Design, Implementation and Performance Analysis of Test Pattern Generator for Built-In Self-Test using m-GDI Technology}

\author{
Y G Praveen Kumar ${ }^{1 *}$, B S Kariyappa ${ }^{2}$, M Z Kurian ${ }^{3}$ \\ 1 Assistant Professor, Department of Electronics \& Communication Engineering, Sri \\ Siddhartha Institute of Technology, SSAHE, Maraluru, 572105, Tumakuru \\ 2 Professor \& Head, Department of ECE, R V College of Engineering, Bengaluru, 560059, \\ Karnataka \\ 3 Professor \& Head, Department of ECE, Sri Siddhartha Institute of Technology, SSAHE, \\ Maraluru, 572105, Tumakuru
}

\section{Abstract}

Background: A Linear Feedback Shift Register (LFSR) is typically used for generating the test patterns in built-in self-test (BIST) as it produces pseudorandom patterns at every clock cycle. These pseudo-random patterns are used as test vectors for testing the VLSI circuits. Objective: The pseudorandom patterns generated by the LFSR exhibit low-correlation among the patterns, this increases the switching activity and power dissipation while testing the VLSI circuit. Thus, to reduce the testing power, modified gate diffusion input (m-GDI) logic based LFSR in $45 \mathrm{~nm}$ technology is proposed in this paper. Methods: The circuits are developed on m-GDI technology using the Cadence virtuoso tool and a spectre simulator is used to carry out the simulation. Findings: Comparative analysis revealed that the delay and power are reduced significantly, for the proposed design when compared to the existing LFSRs in conventional CMOS, GDI and reversible logic. Novelty and applications: In conventional LFSR, an external source is necessary to load the seed value and it dissipates more power. But in the proposed design, the seed value is generated by the circuit itself. This reduces the power and critical path delay. Further a complete zero patterns is not possible in conventional LFSR design. But in proposed design, all zero pattern is plausible. The design obtained from this study can be applied in low-power, high-speed BIST circuits.

Keywords: LFSR; Seed Value; Test Patterns; Built-In-Self-Test; m-GDI

\section{Introduction}

BIST is more suitable for testing the digital circuits as it provides a wide range for lowpower designs. A Linear Feedback Shift Register (LFSR) is generally used in BIST as a test vector generator for spawning the test vectors. During every clock cycle, LFSR generates a random sequence that can be used as test vectors for testing the Circuit- 
Under-Test (CUT). Having carefully selected feedback polynomial, LFSR generates the random patterns which attain high fault coverage in a moderately small run of test patterns. Flip-Flops (FF) are the rudimentary component for realizing LFSR ${ }^{(1)}$. As the FFs have a deterministic operation, the stream of test patterns spawned by the LFSR is entirely governed by its present and preceding value. Furthermore, LFSR produces repeating patterns after it clocked through $2^{\mathrm{n}}-1$ cycles where $\mathrm{n}$ represents the number of flip-flops used in LFSR ${ }^{(2,3)}$. The only input needed by the LFSR to produce the test vectors is the clock signal.

Different techniques and methodologies are proposed for implementing the test pattern generator that aims at reducing power, delay and area. Different logics like transmission gate logic, pass transistor (PT) logic, gate diffusion input (GDI) logic can be used to generate test patterns. PT and GDI technique-based test pattern generators perform better in terms of delay, power and area than the conventional CMOS design ${ }^{(4,5)}$. But these techniques are not compatible with the standard CMOS fabrication process and eventually increase the complexity in the fabrication. With slight modification in the architecture of LFSR, highly random patterns can be achieved ${ }^{(6-8)}$. But this increases the area and complexity with increased length. LFSR based on the bit swapping technique is presented in ${ }^{(9)}$. Bit swapping reduces the power but at the cost of area overhead. Thus, it is observed from the literature that, power, delay and area exhibit the trade-off.

To address these issues, in this research paper m-GDI based LFSR is proposed which reduces the power and delay when compared to conventional CMOS logic.

\section{Basics of $\mathrm{m}$-GDI}

There are many methods to optimize the power. One such method is $\mathrm{m}$-GDI technique. In this technique, a basic m-GDI cell is used to build all the circuit components. m-GDI technique allows us to apply more than one input (G, P and N) unlike CMOS logic where only one input can be applied. This reduces the number of transistors being used for the design and thereby power and area are reduced. The basic m-GDI cell is given in Figure 1.

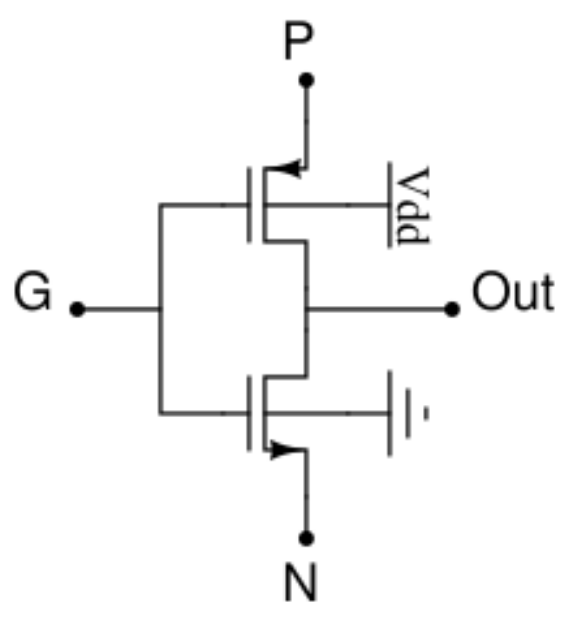

Fig 1. Basic m-GDI cell ${ }^{(10)}$

Modified GDI (m-GDI) cell contains two transistors i.e. NFET and PFET. Gates of NFET and PFET are shorted and it represents the ' $G$ ' terminal of the $\mathrm{m}$-GDI cell. The source terminal of NFET and PFET represent the ' $\mathrm{N}$ 'and 'P' terminals of the $\mathrm{m}$-GDI cell respectively. Also, the bulk of PFET is attached to $\mathrm{V}_{D D}$ and the bulk of NFET is attached to the ground. By applying different inputs and logic values to G, N and P terminals of m-GDI cell many functions can be realized.

Table 1 Illustrates the realization of different Boolean functions by applying different inputs to G, N and P terminals of the basic m-GDI cell. It also presents the transistor count for realizing different functions in CMOS logic and m-GDI logic. These functions entail around 6-12 transistors in CMOS but in m-GDI, it is reduced to almost threefold. 
Table 1. Function table ${ }^{(10-12)}$

\begin{tabular}{|c|c|c|c|c|c|c|}
\hline \multirow{2}{*}{$\mathbf{N}$} & \multirow{2}{*}{$\mathbf{P}$} & \multirow{2}{*}{ G } & \multirow{2}{*}{ OUT } & \multirow{2}{*}{ Function } & \multicolumn{2}{|c|}{ No. of Transistor } \\
\hline & & & & & m - GDI & CMOS \\
\hline '0’ & B & A & $\overline{\mathrm{A} B}$ & $\mathrm{Z}_{1}$ & 2 & 6 \\
\hline B & ' 1 ' & A & $\overline{\mathrm{A}}+\mathrm{B}$ & $\mathrm{Z}_{2}$ & 2 & 6 \\
\hline ' 1 ' & B & A & $A+B$ & OR & 2 & 6 \\
\hline B & '0’ & A & A B & AND & 2 & 6 \\
\hline \multirow[t]{2}{*}{$\mathrm{C}$} & B & A & $\overline{\mathrm{A}} \mathrm{B}+\mathrm{AC}$ & MUX & 2 & 12 \\
\hline & B & A & $\mathrm{A} \oplus \mathrm{B}$ & XOR & 4 & 8 \\
\hline
\end{tabular}

\section{Proposed System Design and Implementation}

LFSR should be loaded with an initial value (other than zero) which is referred to as seed value. Conventionally, the seed value is loaded into the LFSR through an external source and during every clock cycle, the output of LFSR will be shifted to the right by one position and the new value will be loaded into the first D-FF based on the characteristic polynomial. But in the proposed LFSR, a NOR gate is used to generate the seed value, thus no external circuit is required to load the seed value. During every clock cycle, the value stored in LFSR is shifted to the right and produces pseudo-random patterns based on the polynomial.

For implementing the proposed LFSR, basic gates such as Inverter, NAND, NOR and XOR gates are designed initially using $\mathrm{m}$-GDI logic which uses less number of transistors and dissipates less power. This is followed by the design of master-slave D flip-flop (MSDFF) and proposed LFSR. All the designs are done in $45 \mathrm{~nm}$ technology node with a supply of $1.2 \mathrm{~V}$.

\subsection{Master-Slave D Flip-Flop (MSDFF)}

DFF is the basic building block for designing the LFSR. Accordingly, m-GDI based DFF using 4 NAND gates and an inverter is developed and verified for its functionality. The transistor count for implementing m-GDI based DFF is 18(4 NAND gates utilize 16 transistors and an inverter uses 2 transistors).

$\mathrm{m}$-GDI based DFF when used to implement LFSR results in an erroneous output because of the race around problem. And hence a master-slave DFF (MSDFF) is implemented using $2 \mathrm{~m}$-GDI based DFF and 1 inverter. Thus the number of transistors entailed to realize MSDFF is $38\left(2^{\star} 18+1^{\star} 2\right)$. Schematic and the layout of $\mathrm{m}$-GDI based MSDFF is shown in Figure 2 and Figure 3 respectively. The total net length used in the layout is $41.85 \mu \mathrm{m}$ and the chip area of the layout is $65.42 \mu \mathrm{m}^{2}$.

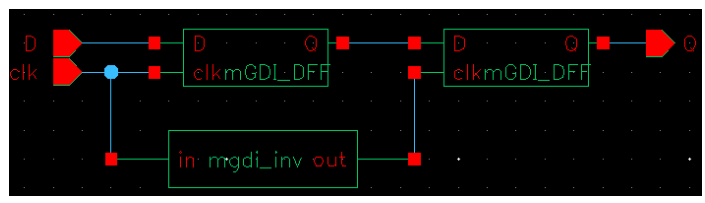

Fig 2. Schematic of m-GDI based MSDFF

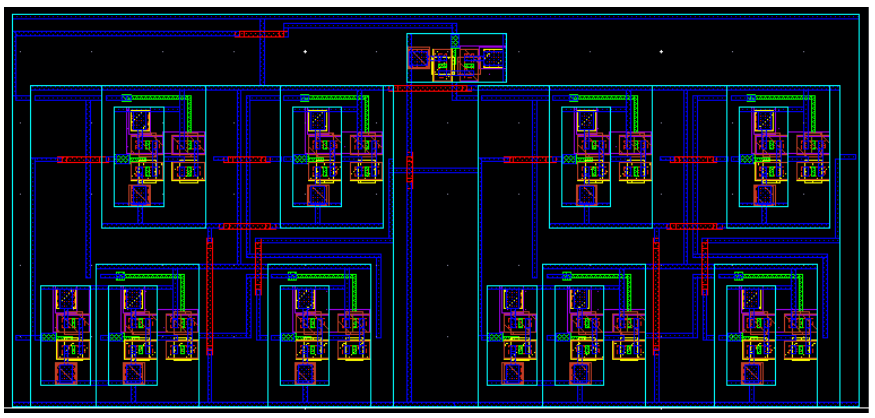

Fig 3. Layout of $m-G D I$ based MSDFF 


\subsection{Proposed LFSR}

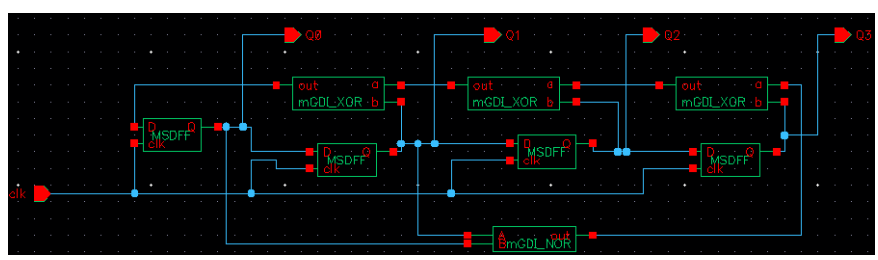

Fig 4. Schematic of Proposed LFSR

Figure 4 illustrates the circuit of 4 -bit LFSR using a self-seeding scheme. It includes 4 MSDFF, 3 XOR gates and 1 NOR gate. Thus a total of $168\left(4{ }^{\star} 38+3{ }^{\star} 4+1{ }^{\star} 4\right)$ transistors are entailed. The feedback polynomial chosen for implementing LFSR is $\mathrm{x}^{4}$ $+\mathrm{x}^{3}+\mathrm{x}^{2}+1$. The bit values that affect the subsequent state of LFSR are called the taps. The taps are XOR'd successively with the output value and then fed back to the first MSDFF as shown in Figure 4. It works on the falling edge of the clock viz. the value of the output bits is shifted to right at every negative edge of the clock and a new value is loaded into the first MSDFF which is determined by the output of NOR gate, $4^{\text {th }}, 3^{\text {rd }}$ and $2^{\text {nd }}$ MSDFFs. The input $\mathrm{D}_{0}$ to the first MSDFF during every clock cycle can be expressed as Equation (1):

$$
\mathrm{D}_{0}(\mathrm{n})=\mathrm{Q}_{3}(\mathrm{n}-1) \text { xor } \mathrm{Q}_{2}(\mathrm{n}-1) \text { xor } \mathrm{Q}_{1}(\mathrm{n}-1) \text { xor }\left\{\mathrm{Q}_{1}(\mathrm{n}-1) \text { nor } \mathrm{Q}_{0}(\mathrm{n}-1)\right\}
$$

Figure 5 illustrates the layout of the proposed 4-bit LFSR. The chip area of the layout is $645.63 \mu \mathrm{m}^{2}$ and the length of the wire used for developing the layout $362.21 \mu \mathrm{m}$. The layouts in Figure 3 and Figure 5 are optimized in area without violating any design rules.

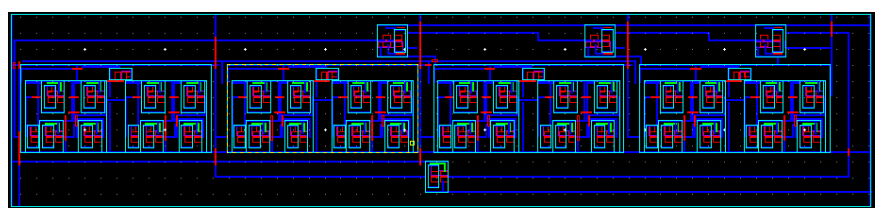

Fig 5. Layout of proposed LFSR

\section{Results and Discussion}

All the designs are done in $45 \mathrm{~nm}$ Technology using Cadence virtuoso tool and functional verification is done using a spectre simulator at $\mathrm{V}_{D D}=1.2 \mathrm{~V}$.

Figure 6 represents the simulation waveform of 4-bit LFSR based on m-GDI technology. At every negative edge of the clock, the output bits $\left(\mathrm{Q}_{0}\right.$ to $\left.\mathrm{Q}_{3}\right)$ of MSDFF are shifted to the right and the feedback mechanism loads the new value into the left-most MSDFF which represents the $\mathrm{Q}_{0}$ bit. The last signal in Figure 6 (red colored signal) is the power signal from which peak power and total power is estimated.

Table 2. Performance metric of proposed LFSR

\begin{tabular}{lll}
\hline & Schematic & Layout \\
\hline Delay(psec) & 144.37 & 476.93 \\
Peak Power $(\mu \mathbf{w})$ & 119.13 & 167.22 \\
Dyn. Power(nW) & 227.05 & 439.55 \\
Static Power(nW) & 4.147 & 4.147 \\
Total Power(nW) & 231.2 & 443.7 \\
\hline
\end{tabular}

The performance metric of the proposed LFSR in schematic and layout is given in Table 2. From the table, it is evident that the delay and power values are slightly more in layout (AV Extracted view) than the schematic because of parasitic resistance and capacitance. 


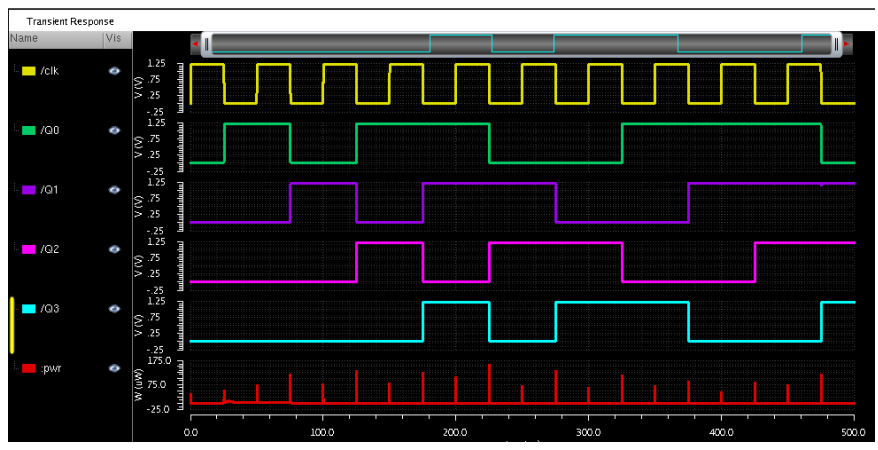

Fig 6. Simulation waveform of proposed LFSR

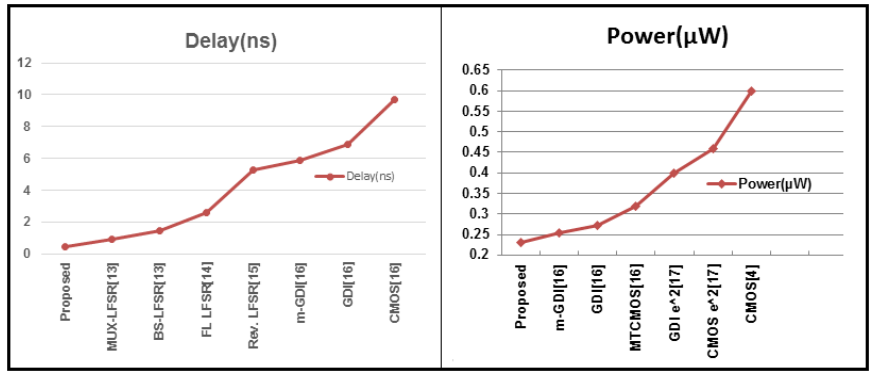

Fig 7. Delay and Power comparison of proposed LFSR with existing designs. Footnote: FL-LFSR: Fuzzy Logic based LFSR, BS-LFSR: Bit Swapping LFSR, MUX-LFSR: MUX based LFSR, Rev-LFSR: Reversible Logic based LFSR, GDI-LFSR: GDI based LFSR.

Figure 7 illustrates the delay and power analysis of the proposed design with existing literature. The critical delay in the proposed design is reduced by $67.56 \%, 81.75 \%, 90.94 \%, 91.87 \%, 93 \%$ and $95.06 \%$ when compared with LFSRs using bitswapping technique $^{(13)}$, fuzzy logic ${ }^{(14)}$, reversible logic ${ }^{(15)}, \mathrm{m}$-GDI logic ${ }^{(16)}$, GDI logic ${ }^{(16)}$ and conventional CMOS logic ${ }^{(16)}$. In the same way, power is also reduced by $9.8 \%, 43.9 \%$, and $61.6 \%$ in proposed LFSR when compared to the $\mathrm{m}-\mathrm{GDI}$, GDI and CMOS based LFSRs presented in articles ${ }^{(16,17)}$ and ${ }^{(4)}$ respectively. Furthermore, the chip area in the proposed m-GDI based LFSR is reduced by $63 \%$ when compared with GDI based LFSR proposed in ${ }^{(17)}$.

\section{Conclusion}

This study presents an implementation of 4-bit LFSR using m-GDI technology in 45nm technology. Conventionally initial value other than logic- 0 needs to be loaded into the LFSR to generate pseudo-random patterns. But in the proposed LFSR, the seed value is generated by the LFSR itself. The results obtained have proved that the delay and power in the proposed design are reduced by $95.06 \%$ and $61.6 \%$ respectively when compared to the conventional CMOS based designs.

Thus, the proposed LFSR can be used to implement the BIST in high-speed and low-power applications, as m-GDI based design reduces the critical delay and dissipates less power than conventional CMOS logic. Also, it can be used to devise a parallel sequence generator in Cryptology applications.

\section{Acknowledgement}

This work is carried out in Cadence lab at Sri Siddhartha Institute of Technology, Karnataka, India and is supported by SSAHE, A deemed to be University, Karnataka, India. Also, the technical support is offered by the faculty of ECE, R V College of Engineering, Karnataka India. Authors have expressed their earnest thanks to the faculty of SSIT and RVCE for their extended support to carry out this work.

\section{References}

1) Hassan S, Bokhari MU. Design of Pseudo Random Number Generator using Linear Feedback Shift Register. International Journal of Engineering and Advanced Technology. 2019;9(2):1956-1965. 
2) Akhila K, Karuna N, Shirur YJM. Design and Implementation of Power Efficient Logic BIST With High Fault Coverage Using Verilog. 2018 International Conference on Networking, Embedded and Wireless Systems (ICNEWS). 2018;p. 1-6. Available from: https://doi.org/10.1109/ICNEWS.2018.8903923.

3) Jayasanthi M, Kowsalyadevi AK. Low Power Implementation of Linear Feedback Shift Registers. International Journal of Recent Technology and Engineering. 2019;8(2):2375-2379. Available from: https://www.ijrte.org/wp-content/uploads/papers/v8i2/A3379058119.pdf.

4) Singh SK, Gupta MD, Mani S, Chauhan RK. Design of LFSR Circuit based on High Performance XOR gate. International Conference on Electrical and Electronics Engineering. 2020;p. 656-660. Available from: https://doi.org/10.1109/ICE348803.2020.9122875.

5) Mohan M, Pillai SS. Review on LFSR for Low Power BIST. 2019 3rd International Conference on Computing Methodologies and Communication (ICCMC). 2019;p. 873-876. Available from: https://doi.org/10.1109/ICCMC.2019.8819698.

6) Durga R, Rashmika C, Madhumitha O, Suvetha. Design and Synthesis of LFSR based Random Number Generator. 3rd International Conference on Smart Systems and Inventive Technology. 2020;p. 438-442. Available from: https://doi.org/10.1109/ICSSIT48917.2020.9214240.

7) Dilip PS, Somanathan GR, Bhakthavatchalu R. Reseeding LFSR for Test Pattern Generation. International Conference on Communication and Signal Processing. 2019;p. 921-925. Available from: https://doi.org/10.1109/ICCSP.2019.8698025.

8) Tan Z, Guo W, Gong G, Lu H. A New Pseudo-Random Number Generator Based On The Leap-Ahead LFSR Architecture. 2018 IEEE International Conference on Integrated Circuits, Technologies and Applications (ICTA). 2018;p. 57-58. Available from: https://doi.org/10.1109/CICTA.2018.8706101.

9) Bagalkoti A, Shirol SB, Kumar P. Design and Implementation of 8-bit LFSR, Bit-Swapping LFSR and Weighted Random Test Pattern Generator: A Performance Improvement. 2019 International Conference on Intelligent Sustainable Systems (ICISS). 2019;p. 82-86. Available from: https://doi.org/10. 1109/ISS1.2019.8908063.

10) Smitha GS, Aradhya HR. m-GDI based parallel adder for low power applications. Microsystem Technologies. 2019;25:1653-1658. Available from: https://ink.springer.com/article/10.1007/s00542-017-3438-1.

11) Balaji SR, Manikandan DR, and VV. Scaling Down Area - Delay - Power Efficient Of Carry Select Adder Using Gdi. International Journal of Engineering Research and. 2018;V6(02):1-4. Available from: https://dx.doi.org/10.17577/ijertcon039.

12) Kumar YGP, Kariyappa BS, Shashank SM, Bharath CN. Performance Analysis of Multipliers Using Modified Gate Diffused Input Technology. IETE Journal of Research. 2020;p. 1-13. Available from: https://dx.doi.org/10.1080/03772063.2020.1782778. doi:10.1080/03772063.2020.1782778.

13) Murugan SV, Sathiyabhama B. Bit-swapping linear feedback shift register (LFSR) for power reduction using pre-charged XOR with multiplexer technique in built in self-test. Journal of Ambient Intelligence and Humanized Computing. 2021;12(6):6367-6373. Available from: https://dx.doi.org/10.1007/s12652020-02222-5.

14) Kumar G, Saminadan. Low Power LFSR for BIST Applications. 2nd International Conference on Intelligent Computing and Control Systems. 2018;p. 1979-1984. Available from: https://doi.org/10.1109/ICCONS.2018.8663184.

15) Reddy BNK, Reddy GSV, Vani BV. Design and Implementation of an Efficient LFSR using 2-PASCL and Reversible Logic Gates. IEEE Bombay Section Signature Conference. 2020;p. 247-250. Available from: https://doi.org/10.1109/IBSSC51096.2020.9332213.

16) Oommen R, George MK, Joseph S. Study and Analysis of Various LFSR Architectures. International Conference on Circuits and Systems in Digital Enterprise Technology (ICCSDET). 2018;p. 1-6. Available from: https://doi.org/10.1109/ICCSDET.2018.8821227.

17) Suman JV, Jyothi P, Vaani. Yanamandra Ravi Sekhar. Design of Low Power and Area Efficient Hybrid Linear Feedback Shift Register. Journal of Critical Reviews. 2020;7(11):2333-2345. Available from: https://www.bibliomed.org/mnsfulltext/197/197-1595580964.pdf?1635234528. 